\title{
Pervasive Detection of Process Races in Deployed Systems
}

\author{
Oren Laadan, Nicolas Viennot, Chia-Che Tsai, \\ Chris Blinn, Junfeng Yang, and Jason Nieh \\ orenl@cs.columbia.edu, nviennot@cs.columbia.edu, chiache.tsai@gmail.com, \\ cpb2114@columbia.edu, junfeng@cs.columbia.edu, nieh@cs.columbia.edu \\ Department of Computer Science \\ Columbia University
}

\begin{abstract}
Process races occur when multiple processes access shared operating system resources, such as files, without proper synchronization. We present the first study of real process races and the first system designed to detect them. Our study of hundreds of applications shows that process races are numerous, difficult to debug, and a real threat to reliability. To address this problem, we created RACEPRO, a system for automatically detecting these races. RACEPRO checks deployed systems in-vivo by recording live executions then deterministically replaying and checking them later. This approach increases checking coverage beyond the configurations or executions covered by software vendors or beta testing sites. RACEPRO records multiple processes, detects races in the recording among system calls that may concurrently access shared kernel objects, then tries different execution orderings of such system calls to determine which races are harmful and result in failures. To simplify race detection, RACEPRO models under-specified system calls based on load and store micro-operations. To reduce false positives and negatives, RACEPRO uses a replay and go-live mechanism to distill harmful races from benign ones. We have implemented RACEPRO in Linux, shown that it imposes only modest recording overhead, and used it to detect a number of previously unknown bugs in real applications caused by process races.
\end{abstract}

\section{Categories and Subject Descriptors:}

D.2.4 [Software Engineering]: Software/Program Verification; D.4.5 [Operating Systems]: Reliability

\section{General Terms:}

Design, Reliability, Verification

\section{Keywords:}

Record-replay, Debugging, Race Detection, Model Checking

Permission to make digital or hard copies of all or part of this work for personal or classroom use is granted without fee provided that copies are not made or distributed for profit or commercial advantage and that copies bear this notice and the full citation on the first page. To copy otherwise, to republish, to post on servers or to redistribute to lists, requires prior specific permission and/or a fee.

SOSP '11, October 23-26, 2011, Cascais, Portugal.

Copyright (C) 2011 ACM 978-1-4503-0977-6/11/10 ... \$10.00.

\section{Introduction}

While thread races have drawn much attention from the research community $[9,11,30,36,38]$, little has been done for process races, where multiple processes access an operating system (OS) resource such as a file or device without proper synchronization. Process races are much broader than time-of-check-to-time-of-use (TOCTOU) races or signal races [39]. A typical TOCTOU race is an atomicity violation where the permission check and the use of a resource are not atomic, so that a malicious process may slip in. A signal race is often triggered when an attacker delivers two signals consecutively to a process to interrupt and reenter a non-reentrant signal handler. In contrast, a process race may be any form of race. Some real examples include a shutdown script that unmounts a file system before another process writes its data, ps I grep X shows $N$ or $N+1$ lines depending on the timing of the two commands, and make $-\mathrm{j}$ failures.

To better understand process races, we present the first study of real process races. We study hundreds of real applications across six Linux distributions and show that process races are numerous and a real threat to reliability and security. For example, a simple search on Ubuntu's software management site [2] returns hundreds of process races. Compared to thread races that typically corrupt volatile application memory, process races are arguably more dangerous because they often corrupt persistent and system resources. Our study also reveals that some of their characteristics hint towards potential detection methods.

We then present RACEPRO, the first system for automatically detecting process races beyond TOCTOU and signal races. RACEPRO faces three key challenges. The first is scope: process races are extremely heterogeneous. They may involve many different programs. These programs may be written in different programming languages, run within different processes or threads, and access diverse resources. Existing detectors for thread or тостоU races are unlikely to work well with this heterogeneity.

The second challenge is coverage: although process races are numerous, each particular process race tends to be highly elusive. They are timing-dependent, and tend to surface only in rare executions. Arguably worse than thread races, they may occur only under specific software, hardware, and user configurations at specific sites. It is hopeless to rely on a few software vendors and beta testing sites to create all possible configurations and executions for checking.

The third challenge is algorithmic: what race detection algorithm can be used for detecting process races? Existing algorithms assume well-defined load and store instructions and thread synchronization primitives. However, the effects of system calls are often under-specified and process synchronization primitives are very 
different from those used in shared memory. For instance, what shared objects does execve access? In addition to reading the inode of the executed binary, an obvious yet incomplete answer, execve also conceptually writes to /proc, which is the root cause of the ps I grep X race (\$5). Similarly, a thread-join returns only when the thread being waited for exits, but wait may return when any child process exits or any signal arrives. Besides fork-wait, processes can also synchronize using pipes, signals, ptrace, etc. Missing the (nuanced) semantics of these system calls can lead to false positives where races that do not exist are mistakenly identified and, even worse, false negatives where harmful races are not detected.

RACEPRO addresses these challenges with four ideas. First, it checks deployed systems in-vivo. While a deployed system is running, RACEPRO records the execution without doing any checking. RACEPRO then systematically checks this recorded execution for races offline, when the deployed system is idle or by replicating the execution to a dedicated checking machine. By checking deployed systems, RACEPRO mitigates the coverage challenge because all user machines together can create a much larger and more diverse set of configurations and executions for checking. Alternatively, if a configuration or execution never occurs, it is probably not worth checking. By decoupling recording and checking [7], RACEPRO reduces its performance overhead on the deployed systems.

Second, RACEPRO records a deployed system as a system-wide, deterministic execution of multiple processes and threads. RACEPRO uses lightweight OS mechanisms developed in our previous work [17] to transparently and efficiently record nondeterministic interactions such as related system calls, signals, and shared memory accesses. No source code or modifications of the checked applications are required, mitigating the scope challenge. Moreover, since processes access shared OS resources through system calls, this information is recorded at the OS level so that RACEPRO can use it to detect races regardless of higher level program semantics.

Third, to detect process races in a recorded execution, RACEPRO models each system call by what we call load and store microoperations to shared kernel objects. Because these two operations are well-understood by existing race detection algorithms, RACEPRO can leverage these algorithms, mitigating the algorithmic challenge. To reduce manual annotation overhead, RACEPRO automatically infers the micro-operations a system call does by tracking how it accesses shared kernel objects, such as inodes. Given these micro-operations, RACEPRO detects load-store races when two concurrent system calls access a common kernel object and at least one system call stores to the object. In addition, it detects waitwakeup races such as when two child processes terminate simultaneously so that either may wake up a waiting parent. To our knowledge, no previous algorithm directly handles wait-wakeup races.

Fourth, to reduce false positives and negatives, RACEPRO uses replay and go-live to validate detected races. A race detected based on the micro-operations may be either benign or harmful, depending on whether it leads to a failure, such as a segmentation fault or a program abort. RACEPRO considers a change in the order of the system calls involved in a race to be an execution branch. To check whether this branch leads to a failure, RACEPRO replays the recorded execution until the reordered system calls then resumes live execution. It then runs a set of built-in or user-provided checkers on the live execution to detect failures, and emits a bug report only when a real failure is detected. By checking many execution branches, RACEPRO reduces false negatives. By reporting only harmful races, it reduces false positives.

We have implemented RACEPRO in Linux as a set of kernel components for record, replay, and go-live, and a user-space explo- ration engine for systematically checking execution branches. Our experimental results show that RACEPRO can be used in production environments with only modest recording overhead, less than $2.5 \%$ for server and $15 \%$ for desktop applications. Furthermore, we show that RACEPRO can detect 10 real bugs due to process races in widespread Linux distributions.

This paper is organized as follows. $\$ 2$ presents a study of process races and several process race examples. $\$ 3$ presents an overview of the RACEPRO architecture. $\$ 4$ describes the execution recording mechanism. $\$ 5$ describes the system call modeling using micro-operations and the race detection algorithm. \$6 describes how replay and go-live are used to determine harmful races. $\S 7$ presents experimental results. $\S 8$ discusses related work. Finally, $\S 9$ presents some concluding remarks and directions for future work.

\section{Process Race Study}

We conducted a study of real process races with two key questions in mind. First, are process races a real problem? Second, what are their characteristics that may hint towards how to detect them? We collected bugs from six widespread Linux distributions, namely Ubuntu, RedHat, Fedora, Gentoo, Debian, and CentOS. For each distribution, we launched a search query of "race" on the distribution's software management website. We manually examined a random sample of the returned pages, identified all unique bugs in the sampled pages, and classified these bugs based on whether they resulted in process or thread races. Raw data of the studied bugs is available [1]. $\$ 2.1$ presents our findings. $\$ 2.2$ describes four process race examples from the most serious to the least.

\subsection{Findings}

Table 1 summarizes the collected pages and bugs; Fedora and Redhat results are combined as they share the same management website. For each distribution, we show the number of pages returned for our query (Returned), the number of pages sampled and manually examined (Sampled), the number of process races (Process) and the subset of which were TOCTOU races, the number of thread races (Thread), and the total number of bugs in the sampled pages (Total).

Process races are numerous. Of the 150 sampled bugs, 109 resulted in process races, a dominating majority; the other 41 bugs resulted in thread races. However, thread races are likely underrepresented because the websites we searched are heavily used by Linux distribution maintainers, not developers of individual applications. Of the 109 process races, 84 are not TOCTOU races and therefore cannot be detected by existing TостоU detectors. Based on this sample, the 7,498 pages that our simple search returned may extrapolate to over 1,500 process races. Note that our counting is very conservative: the sampled pages contain an additional 58 likely process races, but the pages did not contain enough information for us to understand the cause, so we did not include them in Table 1.

\begin{tabular}{c|cc|ccc} 
& \multicolumn{2}{|c|}{ Pages } & \multicolumn{3}{c}{ Bugs } \\
Distribution & Returned & Sampled & Total & Process & Thread \\
\hline Ubuntu & 3330 & 300 & 45 & $42(1)$ & 3 \\
Fedora/RedHat & 1070 & 100 & 52 & $30(10)$ & 22 \\
Gentoo & 2360 & 60 & 31 & $23(10)$ & 8 \\
Debian & 768 & 40 & 17 & $12(4)$ & 5 \\
CentOS & 1500 & 40 & 5 & $2(0)$ & 3 \\
\hline Total & 9028 & 540 & 150 & $109(25)$ & 41
\end{tabular}

Table 1: Summary of collected pages and bugs. 


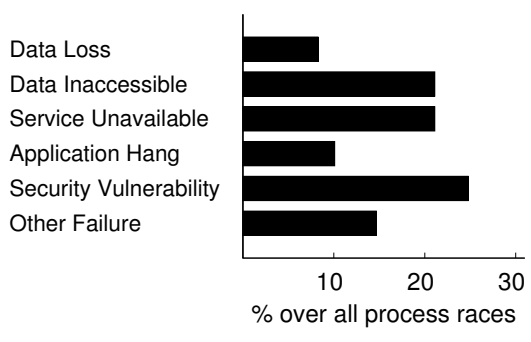

Figure 1: Process races breakdown by effects.

Process races are dangerous. Compared to thread races that typically corrupt volatile application memory, process races are arguably more dangerous because they often corrupt persistent and system resources. Indeed, the sampled process races caused security breaches, files and databases to become corrupted, programs to read garbage, and processes to get stuck in infinite loops. Figure 1 summarizes the effects of all process races from Table 1.

Process races are heterogeneous. The sampled process races spread across over 200 programs, ranging from server applications such as MySQL, to desktop applications such as OpenOffice, to shell scripts in Upstart [4], an event-driven replacement of System $\mathrm{V}$ init scripts. Figure 2 breaks down the process races by packages, processes, and programming languages involved. Over half of the 109 process races, including all examples described in $\$ 2.2$, require interactions of at least two programs. These programs are written in different programming languages such as C, Java, PHP, and shell scripts, run in multiple processes, synchronize via fork and wait, pipes, sockets, and signals, and access resources such as files, devices, process status, and mount points.

This heterogeneity makes it difficult to apply existing detection methods for thread races or TOCTOU races to process races. For instance, static thread race detectors [11] work only with one program written in one language, and dynamic thread race detectors [38] work only with one process. To handle this heterogeneity, RACEPRO's race detection should be system-wide.

Process races are highly elusive. Many of the process races, including Bug 1 and 3 described in $\$ 2.2$, occur only due to sitespecific software, hardware, and user configurations. Moreover, many of the sampled process races, including all of those described in $\$ 2.2$, occur only due to rare runtime factors. For example, Bug 1 only occurs when a database shutdown takes longer than usual, and Bug 2 only occurs when a signal is delivered right after a child process exited. These bugs illustrate the advantage of checking deployed systems, so that we can rely on real users to create the diverse configurations and executions to check.

Process race patterns. Classified by the causes, the 109 process races fall into two categories. Over two thirds (79) are execution order violations [20], such as Bug 1, 3, and 4 in $\$ 2.2$, where a set of events are supposed to occur in a fixed order, but no synchronization operations enforce the order. Less than one third (30) are atomicity violations, including all TOCTOU bugs; most of them are the simplest load-store races, such as Bug 2 in $\$ 2.2$. Few programs we studied use standard locks (e.g., flock) to synchronize file system accesses among processes. These patterns suggest that a lockset-based race detection algorithm is unlikely to work well for detecting process races. Moreover, it is crucial to use an algorithm that can detect order violations

\subsection{Process Race Examples}

Bug 1: Upstart-MySQL. mysqld does not cleanly terminate during system shutdown, and the file system becomes corrupted. This failure is due to an execution order violation where S20sendsigs, the shutdown script that terminates processes, does not wait long enough for MySQL to cleanly shutdown. The script then fails to unmount the file system which is still in use, so it proceeds to reboot the system without cleanly unmounting the file system. Its occurrence requires a combination of many factors, including the mixed use of Systems V initialization scripts and Upstart, a misconfiguration so that S20sendsigs does not wait for daemons started by Upstart, insufficient dependencies specified in MySQL's Upstart configuration file, and a large MySQL database that takes a long time to shut down.

Bug 2: dash-MySQL. The shell wrapper mysql_safe of the MySQL server daemon mysqld goes into an infinite loop with $100 \%$ CPU usage after a MySQL update. This failure is due to an atomicity violation in dash, a small shell Debian uses to run daemons [3]. It occurs when dash is interrupted by a signal unexpectedly. Figure 3 shows the event sequence causing this race. To run a new background job, dash forks a child process and adds it to the job list of dash. It then calls setjmp to save an execution context and waits for the child to exit. After the child exits, wait returns, and dash is supposed to remove the child from the job list. However, if a signal is delivered at this time, dash's signal handler will call longjmp to go back to the saved context, and the subsequent wait call will fail because the child's exit status has been collected by the previous wait call. The job list is still not empty, so dash gets stuck waiting for the nonexistent child to exit. Although this bug is in dash, it is triggered in practice by a combination of dash, the mysql_safe wrapper, and mysqld.

Bug 3: Mutt-OpenOffice. OpenOffice displays garbage when a user tries to open a Microsoft (MS) Word attachment in the Mutt mail client. This failure is due to an execution order violation when mutt prematurely overwrites the contents of a file before OpenOffice uses this file. It involves a combination of Mutt, OpenOffice, a user configuration entry in Mutt, and the openoffice shell script wrapper. The user first configures Mutt to use the openoffice
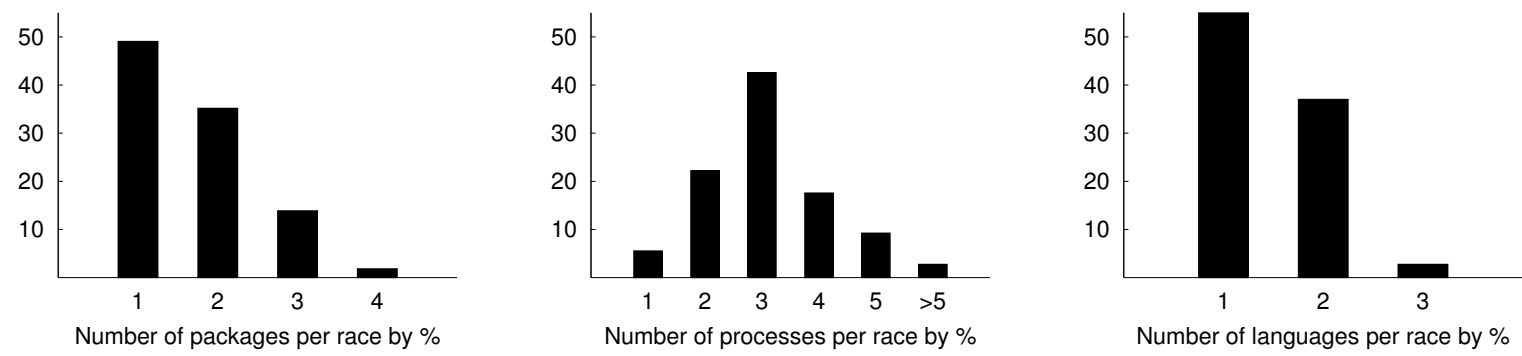

Figure 2: Process races breakdown. X axis shows the number of software packages, processes, or programming languages involved. Y axis shows the percentage of process races that involve the specific number of packages, processes, or languages. To avoid inflating the number of processes, we count a run of a shell script as one process. (Each external command in a script causes a fork.) 


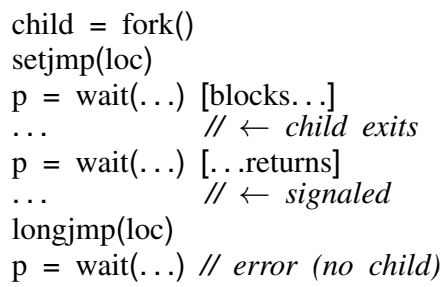

Figure 3: dash-MySQL race.

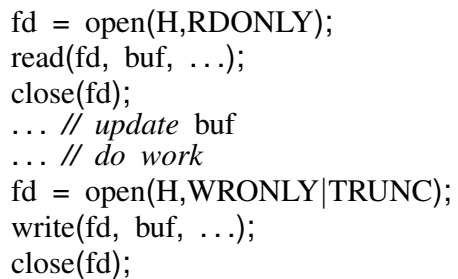

Figure 4: bash race. wrapper to open MS Word attachments. To show an attachment, mutt saves the attachment to a temporary file, spawns the configured viewer in a new process, and waits for the viewer process to exit. The openoffice wrapper spawns the actual OpenOffice binary and exits at once. mutt mistakes this exit as the termination of the actual viewer, and overwrites the temporary file holding the attachment with all zeros, presumably for privacy reasons.

Bug 4: bash. The bash shell history is corrupted. This failure is due to an atomicity violation when multiple bash shells write concurrently to .bash_history without synchronization. When bash appends to the history file, it correctly uses 0_APPEND. However, it also occasionally reads back the history file and overwrites it, presumably to keep the history file under a user-specified size. Figure 4 shows this problematic sequence of system calls. bash also runs this sequence when it exits. When multiple bash processes exit at the same time, the history file may be corrupted.

\section{Architecture Overview}

RACEPRO is designed to automatically detect process races using the workflow shown in Figure 5. It consists of three steps, the first of which runs on the deployed system, while the latter two can run elsewhere on a separate replay system to avoid any performance impact on the deployed system. First, a recorder records the execution of a deployed system while the system is running and stores the recording in a log file. Second, an explorer reads the log and detects load-store and wait-wakeup races in the recorded execution. Third, each race is validated to determine if it is harmful. An execution branch of the recorded execution corresponding to each race is computed by systematically changing the order of system calls involved in the race. For each execution branch, a modified log is constructed that is used to replay execution with the changed order of system calls. A replayer replays the respective modified log up to the occurrence of the race, then causes it to resume live execution from that point onward. A set of built-in and user-provided checkers then check whether the execution results in misbehavior or a failure such as a segmentation fault. By examining the effects of a live execution, we distinguish harmful races from false or be-

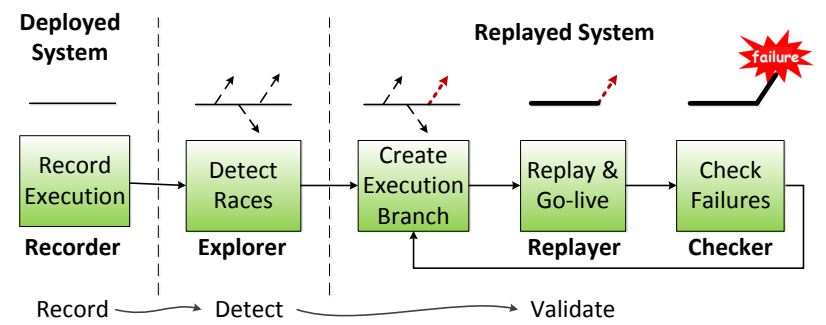

Figure 5: RACEPRO Workflow. Thin solid lines represent recorded executions; thick solid lines represent replayed executions. Dashed arrows represent potentially buggy execution branches. The dotted thick arrow represents the branch RACEPRO selects to explore. nign ones, thus reducing false positives $[25,30]$. The live part of the re-execution is also recorded, so that users can deterministically replay detected bugs for debugging.

Figure 6 shows the RACEPRO architecture used to support its workflow. Of the four main architectural components, the recorder and the replayer run in kernel-space, and the explorer and checkers run in user-space. We will describe how RACEPRO records executions $(\S 4)$ and detects $(\S 5)$ and validates $(\S 6)$ races using these components.

\section{Recording Executions}

RACEPRO's record-replay functionality builds on our previous work on lightweight OS-level deterministic replay on multiprocessors [17]. This approach provides four key benefits for detecting process races. First, RACEPRO's recorder can record the execution of multiple processes and threads with low overhead on a deployed system so that the replayer can later deterministically replay that execution. This makes RACEPRO's in-vivo checking approach possible by minimizing the performance impact of recording deployed systems. Second, RACEPRO's record-replay is applicationtransparent; it does not require changing, relinking, or recompiling applications or libraries. This enables RACEPRO to detect process races that are extremely heterogeneous involving many different programs written in different program languages. Third, RACEPRO's recorder operates at the OS level to log sufficiently fine-grained accesses to shared kernel objects so that RACEPRO's explorer can detect races regardless of high-level program semantics (§5). Finally, RACEPRO's record-replay records executions such that it can later transition from controlled replay of the recording to live execution at any point. This enables RACEPRO to distinguish harmful races from benign ones by allowing checkers to monitor an application for failures ( $\$ 6.2)$.

To record the execution of multiprocess and multithreaded applications, RACEPRO records all nondeterministic interactions between applications and the OS and saves the recording as a log file. We highlight how key interactions involving system calls, signals, and shared memory are handled.

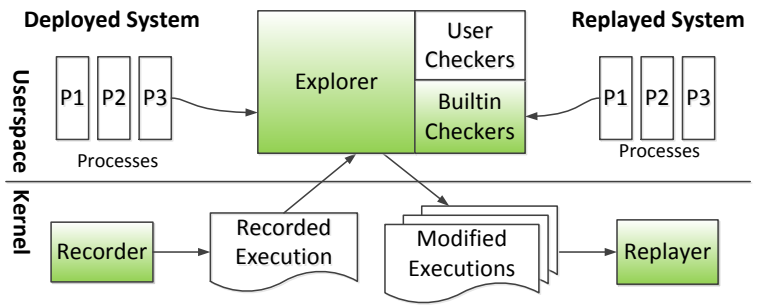

Figure 6: RACEPRO Architecture. Components are shaded. The recorder and the replayer run in kernel-space, and the explorer and the checkers run in user-space. Recorded executions and modified executions are stored in files. 


\begin{tabular}{cl} 
Object & Description \\
\hline inode & file, directory, socket, pipe, tty, pty, device \\
file & file handle of an open file \\
file-table & process file table \\
mmap & process memory map \\
cred & process credentials and capabilities, e.g., user ID \\
global & system-wide properties $(e . g .$, hostname, mounts) \\
pid & process ID (access to process and /proc) \\
ppid & parent process ID (synchronize exit/getppid)
\end{tabular}

Table 2: Shared kernel objects tracked.

System calls. Unlike previous work $[15,31]$ that records and replays a total order of system calls, RACEPRO records and replays a partial order of system calls for speed. RACEPRO enforces no ordering constraints among system calls during record-replay unless they access the same kernel object and at least one of them modifies it, such as a write and a read on the same file. In that case, RACEPRO records the order in the kernel in which the object is accessed by the system calls and later replays the exact same order of accesses. This is done by piggybacking on the synchronization code that the kernel already has for serializing accesses to shared objects. These tracked accesses also help detect process races in a recorded execution (\$5).

Table 2 lists the kernel objects tracked by RACEPro. Most of the entries correspond one-to-one to specific low-level kernel resources, including inodes, files, file-tables, memory maps, and process credentials. The global entry corresponds to system-wide kernel objects, such as the hostname, file system mounts, system time, and network interfaces. For each such system-wide resource there is a unique global kernel object used to track accesses to that resource. The last two entries in the table, pid and ppid, provide a synchronization point to track dependencies on process states. For example, the pid entry of a process is used to track instances where the process is referenced by another process, e.g., through a system call that references the process ID or through the /proc file system. The ppid entry is used to track when an orphan process is re-parented, which is visible through the getppid system call. Both pid and ppid correspond to identifiers that are visible to processes but cannot be modified explicitly by processes.

The recorder only tracks kernel objects whose state is visible to user-space processes, either directly or indirectly. For example, inode state is accessible via the system call lstat, and file-table state is visible through resolving of file descriptor in many system calls. RACEPRO does not track accesses to kernel objects which are entirely invisible to user-space. This avoids tracking superfluous accesses that may pollute the race detection results with unnecessary dependencies. For example, both the fork and exit system calls access the kernel process table, but the order is unimportant to userspace. It only matters that the lifespan of processes is observed correctly, which is already tracked and enforced via the pid resource. If RACEPRO tracked accesses to the kernel process table, it would mistakenly conclude that every two fork system calls are "racy" because they all modify a common resource (\$5). One complication with this approach is that if the kernel object in question controls assignment of identifiers (e.g., process ID in the fork example), it may assign different identifiers during replay because the original order of accesses is not enforced. To address this problem, RACEPRO virtualizes identifiers such as process IDs to ensure the same values are allocated during replay as in the recording.

Signals. Deterministically replaying signals is hard since they must be delivered at the exact same instruction in the target execution flow as during recording. To address this problem, RACE-
PRO uses sync points that correspond to synchronous kernel entries such as system calls. Sending a signal to a target process may occur at any time during the target process's execution. However, RACEPRO defers signal delivery until sync points occur to make their timing deterministic so they are easier to record and replay efficiently. Unlike previous approaches, sync points do not require hardware counters or application modifications, and do not adversely impact application performance because they occur frequently enough in real server and desktop applications due to OS activities.

Shared memory. RACEPRO combines page ownership with sync points to deterministically record and replay the order of shared memory accesses among processes and threads. Each shared memory page is assigned an owner process or thread for some time interval. The owner can exclusively modify that page during the interval and treat it like private memory, avoiding the need to track all memory accesses during such ownership periods. Transitioning page ownership from one process or thread to another is done using a concurrent read, exclusive write (CREW) protocol [10, 19]. To ensure that ownership transitions occur at precisely the same location in the execution during both record and replay, RACEPRO defers such transitions until the owner reaches a sync point. When a process tries to access an owned page, it triggers a page fault, notifies the owner, and blocks until access is granted. Conversely, each owner checks for pending requests at every sync point and, if necessary, gives up ownership. Page faults due to the memory interleaving under the CREW protocol are synchronous kernel entries that deterministically occur on replay and hence are also used as sync points.

\section{Detecting Process Races}

RACEPRO flags a set of system calls as a race if (1) they are concurrent and therefore could have executed in a different order than the order recorded, (2) they access a common resource such that reordering the accesses may change the outcome of the execution. To determine whether a set of system calls are concurrent, RACEPRO constructs a happens-before [18] graph for the recorded execution (§5.1). To determine whether a set of system calls access common resources, RACEPRO obtains the shared kernel resources accessed by system calls from the log file and models the system calls as load and store micro-operations ( $\$ 5.2)$ on those resources. RACEPRO then runs a set of happens-before based race detection algorithms to detect load-store and wait-wakeup races $(§ 5.3)$.

\subsection{The Happens-Before Graph}

We define a partial ordering on the execution of system calls called inherent happens-before relations. We say that system call $S_{1}$ inherently happens-before system call $S_{2}$ if (1) $S_{1}$ accesses some resource before $S_{2}$ accesses that resource, (2) there is a dependency such that $S_{2}$ would not occur or complete unless $S_{1}$ completes, and (3) the dependency must be inferable from the system call semantics. For example, a fork that creates a child process inherently happens-before any system call in the child process, and a write to a pipe inherently happens-before a blocking read from the pipe. On the other hand, there is no inherent happens-before relation between a read and subsequent write to the same file.

RACEPRO constructs the happens-before graph using only inherent happens-before relations, as they represent the basic constraints on the ordering of system calls. Given a recorded execution, RACEPRO constructs a happens-before graph for all recorded system call events by considering pairs of such events. If two events $S_{1}$ and $S_{2}$ occur in the same process and $S_{2}$ is the next system call event that occurs after $S_{1}$, RACEPRO adds a directed edge $S_{1} \rightarrow S_{2}$ in the happens-before graph. If two events $S_{1}$ and $S_{2}$ occur in two 


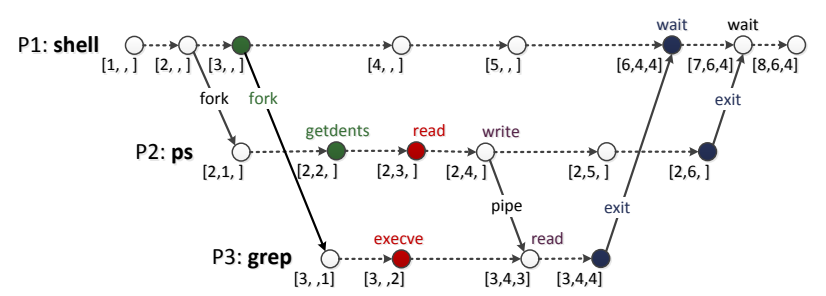

Figure 7: The Happens-before graph and respective vector-clocks (in brackets) for ps I grep $X . P_{i=1,2,3}$ represent the processes involved. The read of process $P_{2}$ and the execve of $P_{3}$ form a load-store race ( $\$ 5.3 .1)$, and so do the second fork of $P_{1}$ and the getdents (read directory entries) of $P_{2}$. The first wait of $P_{1}$ and the exits of $P_{2}$ and $P_{3}$ form a wait-wakeups race (§5.3.2). For clarity, not all system calls are shown.

different processes, RACEPRO adds a directed edge $S_{1} \rightarrow S_{2}$ in four cases:

1. $S_{1}$ is a fork call, and $S_{2}$ is the corresponding fork return in the child process;

2. $S_{1}$ is the exit of a child process, and $S_{2}$ is the corresponding wait in the parent;

3. $S_{1}$ is a kill call, and $S_{2}$ is the corresponding signal delivery in the target process; or

4. $S_{1}$ is a stream (e.g., pipe or socket) write, and $S_{2}$ is a read from the same stream and the data written and the data read overlap.

We say that event $S_{1}$ happens-before $S_{2}$ with respect to a happens-before graph iff there is a directed path from $S_{1}$ to $S_{2}$ in the happens-before graph. Two events are concurrent with respect to a happens-before graph iff neither happens before the other.

RACEPRO also computes the vector-clocks [22] for all the system calls in the happens-before graph. By definition, the vectorclock of $S_{1}$ is earlier than the vector-clock of $S_{2}$ iff $S_{1}$ happensbefore $S_{2}$ with respect to the graph, so comparing the vector-clocks of system calls is a fast and efficient way to test whether they are concurrent.

Our definition of inherent happens-before does not capture all dependencies that may constrain execution ordering. It may be missing happens-before edges that depend on the behavior of the application but cannot be directly inferred from the semantics of the system calls involved. For example, the graph does not capture dependencies between processes via shared memory. It also does not capture dependencies caused by contents written to and read from files. For example, one can implement a fork-join primitive using read and write operations on a file. In some cases, such inaccuracies may make RACEPRO more conservative in flagging racy system calls and thereby identify impossible races. However, such cases will be filtered later by RACEPRO's validation step ( $\$ 6)$ and will not be reported.

Figure 7 shows the happens-before graph for the example command ps I grep X. This command creates two child processes that access grep's entry in the /proc directory: the process that runs grep modifies its command-line data when executed, and the process that runs ps reads that data. A race exists because both processes access the common resource in an arbitrary order, and the end result can be either $N$ or $N+1$ lines depending on that order.

Consider the execve system call in process $P_{3}$ and the read system call in process $P_{2}$. These two system calls are concurrent because there is no directed path between them in the graph. They both access a shared resource, namely, the inode of the file cmd_line in the directory corresponding to $P_{3}$ in /proc. Therefore, these system calls are racy: depending on the precise execu- tion order, read may or may not observe the new command line with the string "X". Similarly, the second fork in process $P_{1}$ and the getdents in process $P_{3}$ are also racy: getdents may or may not observe the newly created entry for process $P_{3}$ in the /proc directory.

In contrast, consider the pipe between $P_{2}$ and $P_{3}$. This pipe is a shared resource accessed by their write and read system calls, respectively. However, these two system calls are not racy because they are not concurrent. There exists a happens-before edge in the graph because a read from the pipe will block until data is available after a write to it.

\subsection{Modeling Effects of System Calls}

Existing algorithms for detecting memory races among threads rely on identifying concurrent load and store instructions to shared memory. To leverage such race detection algorithms, RACEPRO models the effects of a system call on the kernel objects that it may access using two micro-operations: load and store. These microoperations are analogous to the traditional load and store instructions that are well-understood by the existing algorithms, except our micro-operations refer to shared kernel objects, such as inodes and memory maps, instead of an application's real shared memory.

More formally, we associate an abstract memory range with each kernel object. The effect of a system call on a kernel object depends on its semantics. If the system call only observes the object's state, we use a load(obj,range) operation. If it may also modify the object's state, we use a store(obj,range) operation. The argument $o b j$ indicates the affected kernel object, and the argument range indicates the ranges being accessed within that object's abstract memory. A single system call may access multiple kernel objects or even the same kernel object multiple times within the course of its execution.

We use a memory range for a shared kernel object instead of a single memory location because system calls often access different properties of an object or ranges of the object data. For instance, lstat reads the meta-data of files, while write writes the contents of files. They access a common object, but because they access distinct properties of that object, we do not consider them to race. Likewise, read and write system calls to non-overlapping regions in the same file do not race.

Memory ranges are particularly useful to model pathnames. Pathname creation and deletion change the parent directory structure and may race with reading its contents, but pathname creation, deletion, and lookup may only race with each other if given the same pathname. For example, both $\operatorname{creat}(/ \mathrm{tmp} / \mathrm{a})$ and unlink (/tmp/b) may race with a getdents on /tmp, but are unrelated to each other or to an lstat $(/ \mathrm{tmp} / \mathrm{c})$. Modeling all pathname accesses using a single location on the parent directory's inode is too restrictive. Instead, we assign a unique memory location in the parent directory's inode for each possible pathname. We then model pathname creation and deletion system calls as stores to the designated location, pathname lookup system calls as loads from that location, and read directory system calls as loads from the entire pathname space under that directory.

Memory ranges are also useful to model wait system calls which may block on events and wakeup system calls which may trigger events. Example wait and wakeup system calls include wait and exit, respectively, and a blocking read from a pipe and a write to the pipe, respectively. To model the effect of wait and wakeup system calls, we use a special location in the abstract memory of the resource involved. Wait system calls are modeled as loads from that location, and wakeup system calls are modeled as stores to that location. For instance, the exit system call does a store to 


\begin{tabular}{|c|c|c|}
\hline Syscall & Micro-Op & Kernel Object \\
\hline \multirow{5}{*}{ open } & store & file-table \\
\hline & load & inodes of path components \\
\hline & store & inode of directory, if O_CREAT \\
\hline & load & inode of file, if no O_CREAT \\
\hline & store & data of file (range), if O_TRUNC \\
\hline \multirow{4}{*}{ write } & load & process file-table \\
\hline & store & file handle of file \\
\hline & store & inode of file \\
\hline & store & data of file (range) \\
\hline \multirow{5}{*}{ read } & load & process file-table \\
\hline & store & file handle of file \\
\hline & load & inode of file, if regular file \\
\hline & store & inode of file, if a stream \\
\hline & load & data of file (range) \\
\hline \multirow{4}{*}{ getdents } & load & process file-table \\
\hline & store & file handle of directory \\
\hline & load & inode of directory \\
\hline & load & data of directory (range) \\
\hline \multirow{3}{*}{ execve } & load & inodes of path components \\
\hline & store & data of /proc/self/status \\
\hline & store & data of /proc/self/cmdline \\
\hline & load & process memory map \\
\hline clone & store & data of / proc directory \\
\hline \multirow[t]{2}{*}{ exit } & store & 'pid' of self \\
\hline & store & 'ppid' of re-parented children \\
\hline \multirow[t]{2}{*}{ wait } & store & data of /proc directory \\
\hline & load & 'pid' of reaped child \\
\hline getppid & load & 'ppid' of self \\
\hline
\end{tabular}

Table 3: Micro-operations of common system calls.

the special location associated with the parent process ID, and the getppid system call does a load from the same location.

Table 3 shows the template of micro-operations that RACEPRO uses to model nine common system calls: open, write, read, getdents, execve, clone (fork a process), exit, wait, and getppid. The open system call accesses several resources. It stores to the process file-table to allocate a new file descriptor, loads from the inodes of the directories corresponding to the path components, stores to the inode of the parent directory if the file is being created or loads from the file's inode otherwise, and stores to the entire data range of the inode if the file is being truncated.

The write, read, and getdents system calls access three resources: process file-table, file handle, and inode. write loads from the process file-table to locate the file handle, stores to the file handle to update the file position, stores to the meta-data of the file's inode in the file system, and stores to the affected data range of the file's inode. The last two micro-operations both affect the file's inode, but at different offsets. read from a regular file and getdents are similar to write, except that they load from the respective file's or directory's inode. read from a stream, such as a socket or a pipe, is also similar, except that it consumes data and thus modifies the inode's state, so it is modeled as a store to the corresponding inode.

The execve system call accesses several resources. It loads from the inodes of the directories corresponding to the path components. It also stores to the inodes of the status and cmdline files in the /proc directory entry of the process, to reflect the newly executed program name and command line.

The clone, exit, and wait system calls access two resources. clone loads from the process's memory map to create a copy for the newborn child, and stores to the /proc directory inode to reflect the existence of a new entry in it. exit stores to the pid resource of the current process to set the zombie state, and stores to the ppid resource of its children to reparent them to init. wait stores to the reaped child's pid resource to change its state from zombie to dead, and stores to the /proc directory inode to remove the reaped child's entry. RACEPRO detects races between exit and wait based on accesses to the exiting child's pid resource. Similarly, getppid loads from the current process's ppid resource, and RACEPRO detects races between exit and getppid based on accesses to the ppid resource.

To account for system calls that operate on streams of data, such as reads and writes on pipes and sockets, we maintain a virtual write-offset and read-offset for such resources. These offsets are advanced in response to write and read operations, respectively. Consider a stream object with write-offset $L_{W}$ and read-offset $L_{R}$. A write $(f d, b u f, n)$ is modeled as a store to the memory range $\left[L_{W} . . L_{W}+n\right]$ of the object, and also advances $L_{W}$ by $n$. A $\operatorname{read}(\mathrm{fd}, \mathrm{buf}, \mathrm{n})$ is modeled as a load from the memory range $\left[L_{R} . . L_{R}+\tilde{n}\right]$, where $\tilde{n}=\min \left(L_{W}-L_{R}, n\right)$, and also advances $L_{R}$ by $\tilde{n}$.

To account for the effects of signal delivery and handling, we model signals in a way that reflects the possibility of a signal to affect any system call, not just the one system call that was actually affected in the recording. We associate a unique abstract memory location with each signal. A kill system call that sends a signal is modeled as a store to this location. Each system call in the target process is considered to access that location, and therefore modeled as a load from all the signals. This method ensures that any system call that may be affected by a signal would access the shared object that represents that signal.

\subsection{Race Detection Algorithms}

Building on the happens-before graph and the modeling of system calls as micro-operations, RACEPRO detects three types of process races: load-store races (\$5.3.1), wait-wakeups races (\$5.3.2), and wakeup-waits races $(\$ 5.3 .3)$. RACEPRO may also be extended to detect other types of races $(\S 5.3 .4)$.

\subsubsection{Load-Store Races}

A load-store race occurs when two system calls concurrently access the same shared object and at least one is a store operation. In this case, the two system calls could have executed in the reverse order. RACEPRO flags two system calls as a load-store race if (1) they are concurrent; (2) they access the same shared kernel object, and (3) at least one access is a store. In the ps I grep X example shown in Figure 7, the system calls read and execve are flagged as a race because they are concurrent, they access the same resource, and at least one, execve, does a store. In contrast, the system call exit of $P_{3}$ also stores to the same resource, but is not flagged as a race because it is not concurrent with any of them as read happensbefore exit and execve happens-before exit.

RACEPRO detects load-store races using a straightforward happens-before-based race detection algorithm. We chose a happens-before over lockset because processes rarely use standard locks (\$2). RACEPRO iterates through all the shared kernel objects in the recording. For each shared object, it considers the set of all accesses to that object by all system calls, and divides this set into per-process lists, such that the list $L_{i}$ of process $P_{i}$ contains all the accesses performed by that process. RACEPRO now looks at all pairs of processes, $P_{i}, P_{j}, i \neq j$, and considers their accesses to the object. For each access $S_{n} \in L_{i}$, it scans through the accesses $S_{m} \in L_{j}$. If the vector-clocks of $S_{n}$ and $S_{m}$ are concurrent, the pair of system calls is marked as a race. If $S_{n} \rightarrow S_{m}$, then $S_{n} \rightarrow S_{m+k}$, so the scan is aborted and the next access $S_{n+1} \in L_{i}$ 
is considered. If $S_{m} \rightarrow S_{n}$, then $S_{m} \rightarrow S_{n+k}$, so $S_{m+1} \in L_{j}$ is saved so that the next scan of accesses from $L_{j}$ will start from $S_{m+1}$, since we know that earlier events happened-before all remaining accesses in $L_{i}$.

Because system calls may access more than one shared object during their execution, it is possible that the same pair of system calls will be marked more than once. For example, two write system calls from different processes to the same location in the same file will be marked twice, once when the meta-data of the inode is considered, and once when the data of the file is considered. Because RACEPRO detects and later validates $(\$ 6)$ races at the granularity of system calls, it only reports the respective pair of system calls once.

RACEPRO may produce a myriad of races, which can take a long time to produce and later validate. To address this concern, RACEPRO prioritizes which races to examine in two ways. First, RACEPRO may defer or entirely skip races that are less likely to prove harmful, depending on the system calls and resource involved. For example, when analyzing the execution of a parallel compilation, resources related to visual output may be skipped: although many processes may be writing to the standard output, races, if they exist, are likely to be benign. Second, RACEPRO ranks pairs of system calls according to their distance from each other in the happensbefore graph, and examines nearer system calls first.

\subsubsection{Wait-Wakeups Races}

A wait-wakeups race occurs when a wait system call may be woken up by more than a single matching wakeup system call. If the wakeup system calls executed in a different order, the wait system call could have picked a different wakeup than in the original execution. Wait-wakeups races involve at least three system calls. For instance, a wait system call which does not indicate a specific process identifier to wait for will complete if any of its children terminate. Likewise, a blocking read from a stream will complete after any write to the stream.

In these cases, the wait system call essentially uses a wildcard argument for the wakeup condition so that there can be multiple system calls that match the wakeup condition depending on their order of execution. The wait-wakeups race requires a wildcard, otherwise there is only a single matching system call, and thus a single execution order. For instance, a wait system call that requests a specific process identifier must be matched by the exit of that process. In this case, the wait-wakeup relationship implies an inherent happens-before edge in the happens-before graph, since the two system calls must always occur in that order.

RACEPRO flags three system calls as a wait-wakeups race if (1) one is a wait system call, (2) the other two are wakeup system calls that match the wait condition, and (3) the wait system call did not happen-before any of the wakeup system calls. In the ps I grep X example shown in Figure 7, the two exit system calls of $P_{2}$ and $P_{3}$ and the first wait system call of $P_{1}$ are flagged as a wait-wakeups race since both exit calls are concurrent and can match the wait. In contrast, the write and read system calls to and from the pipe are not flagged as a race, because there does not exist a second wakeup system call that matches the read.

RACEPRO detects wait-wakeups races using an algorithm that builds on the load-store race detection algorithm, with three main differences. First, the algorithm considers only those accesses that correspond to wait and wakeup system calls by looking only at locations in the abstract memory reserved for wait and wakeup actions. Second, it considers only pairs of accesses where one is a load and the other is a store, corresponding to one wait and one wakeup system calls. The wait system call must not happen-before the wakeup

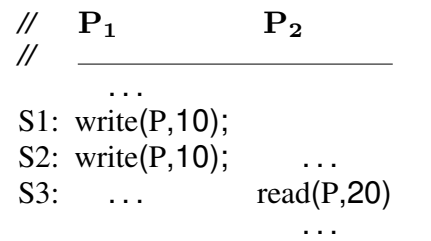

(a)

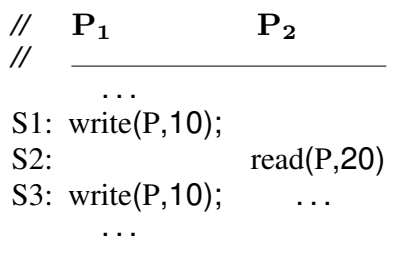

(b)
Figure 8: Wait-wakeups races in streams.

system call. Third, for each candidate pair of wait and wakeup system calls $S_{1}$ and $S_{2}$, RACEPRO narrows its search to the remaining wakeup system calls that match the wait system call by looking for system calls that store to the same abstract memory location. For each matching wakeup system call $S_{3}$, RACEPRO checks whether it would form a wait-wakeups race together with $S_{1}$ and $S_{2}$.

The relative order of the wakeup system calls may matter if their effect on the resource is cumulative. For instance, Figure 8 depicts a cumulative wait-wakeups scenario in which the order of two write system calls to the same stream determines what a matching read would observe. A read from a stream may return less data than requested if the data in the buffer is insufficient. In Figure 8a, a blocking read occurs after two writes and consumes their cumulative data. However, in Figure 8b, the read occurs before the second write and returns the data only from the first write. Note that $S_{2}$ and $S_{3}$ in Figure 8a do not form a load-store race as $S_{2}$ inherently happens-before $S_{3}$. Thus, RACEPRO flags either case as a wait-wakeups race. The relative order of the wakeup system calls does not matter if their effect on the resource is not cumulative, such as with wait and exit system calls.

\subsubsection{Wakeup-Waits Races}

A wakeup-waits race occurs when a wakeup system call may wake up more than a single matching wait system call. Like waitwakeups races, wakeup-waits races involve at least three system calls. For example, a connect system call to a listening socket will wake up any processes which may have a pending accept on that socket; the popular Apache Web server uses this method to balance incoming requests. As another example, a signal sent to a process may interrupt the process during a system call. Depending on the exact timing of events, the signal may be delivered at different times and interrupt different system calls.

Some wakeup system calls only affect the first matching wait system call that gets executed; that system call "consumes" the wakeup and the remaining wait system calls must wait for a subsequent wakeup. Examples include connect and accept system calls, and read and write system calls on streams. In contrast, when two processes monitor the same file using the select system call, a file state change will notify both processes equally. Even in this case, a race exists as the behavior depends on which wait system calls executes first.

RACEPRO flags three system calls as a wakeup-waits race if (1) one is a wakeup system call, (2) the other two are wait system calls that match the wakeup, (3) the wait system calls did not happen-before the wakeup system call. To detect wakeup-waits races, RACEPRO builds on the wait-wakeups race detection algorithm with one difference. For each candidate pair of wait and wakeup system calls $S_{1}$ and $S_{2}$, RACEPRO narrows its search to the remaining wait system calls that match the wakeup system call by looking for system calls that load from the same abstract memory location. For each matching wait system call $S_{3}$, RACEPRO checks whether it would form a wakeup-waits race together with $S_{1}$ and $S_{2}$. 


\subsubsection{Many-System-Calls Races}

RACEPRO's algorithms handle races that involve two system calls for load-store races, and three system calls for both wait-wakeups and wakeup-waits races. However, it is also possible that a race involves more system calls. For example, consider a load-store race that comprises a sequence of four system calls that only if executed in the reverse order, from last to first, will produce a bug. RACEPRO's algorithm will not detect this load-store race since it only considers one pair of system calls at a time. To detect such races, the algorithms can be extended to consider more system calls at a time and more complex patterns of races. An alternative approach is to apply RACEPRO's analysis recursively on modified executions (§6.2).

\section{Validating Races}

A detected process race may be either benign or harmful, depending on whether it leads to a failure. For instance, consider the ps I grep X example again which may output either $N$ or $N+1$ lines. When run from the command line, this race is usually benign since most users will automatically recognize and ignore the difference. However, for applications that rely on one specific output, this race can be harmful and lead to a failure (\$7).

To avoid false positives, RACEPRO validates whether detected races are harmful and reports only harmful races as bugs. For each race, it creates an execution branch in which the racy system calls, which we refer to as anchor system calls, would occur in a different order from the original recorded execution (\$6.1). It replays the modified execution until the race occurs, then makes the execution go-live (\$6.2). It checks the live execution for failures ( 6.3$)$, and, if found, reports the race as a bug.

\subsection{Creating Execution Branches}

RACEPRO does not replay the original recorded execution, but instead replays an execution branch built from the original execution in a controlled way. The execution branch is a truncated and modified version of the original $\log$ file. Given a detected race which, based on its type, involves two or three anchor system calls, RACEPRO creates an execution branch in two steps. First, it copies the sequence of $\log$ events from the original execution recording up to the anchor system calls. Then, it adds the anchor system calls with suitable ordering constraints so that they will be replayed in an order that makes the race resolve differently than in the original recorded execution. The rest of the log events from the original execution are not included in the modified version.

A key requirement in the first step above is that the definition of up to must form a consistent cut [22] across all the processes to avoid deadlocks in replay. A consistent cut is a set of system calls, one from each process, that includes the anchor system calls, such that all system calls and other log events that occurred before this set are on one side of the cut. For instance, if $S_{1}$ in process $P_{1}$ happens-before $S_{2}$ in process $P_{2}$ and we include $S_{2}$ in the consistent cut, then we must also include $S_{1}$ in the cut.

To compute a consistent cut for a set of anchor system calls, RACEPRO simply merges the vector-clocks of the anchor system calls into a unified vector-clock by taking the latest clock value for each process. In the resulting vector-clock, the clock value for each process indicates the last observed happens-before path from that process to any of the anchor system calls. By definition, the source of this happens-before edge is also the last system call of that process that must be included in the cut. For instance, the unified vector-clock for the read and execve race in Figure 7 is $[3,3,2]$, and the consistent cut includes the second fork of $P_{1}$, read of $P_{2}$, and execve of $P_{3}$.
Given a consistent cut, RACEPRO copies the log events of each process, except the anchor system calls, until the clock value for that process is reached. It then adds the anchors in a particular order. For load-store races, there are two anchor system calls. To generate the execution branch, RACEPRO simply flips the order of the anchors compared to the original execution; it first adds the system call that occurred second in the original execution, followed by the one that occurred first. It also adds an ordering constraint to ensure that they will be replayed in that order.

For wait-wakeups races, there are three anchor system calls: two wakeup system calls and a wait system call. To generate the execution branch, RACEPRO first adds both wakeup system calls, then adds a modified version of the wait system call in which its wildcard argument is replaced with a specific argument that will match the wakeup system call that was not picked in the original execution. For example, consider a race with two child processes in exit, either of which may wake up a parent process in wait. RACEPRO first adds both exit system calls, then the wait system call modified such that its wildcard argument is replaced by a specific argument that will cause this wait to pick the exit of the child that was not picked in the original execution. It also adds a constraint to ensure that the parent will execute after that child's exit. The other child is not constrained.

For wakeup-waits races, there are also three anchor system calls: one wakeup system call and two wait system calls. To generate the execution branch, RACEPRO simply flips the order of the two wait system calls compared to the original execution. Races that involve signals, which may be delivered earlier or later than in the original execution, are handled differently. To generate an execution branch for a signal to be delivered earlier, RACEPRO simply inserts the signal delivery event at an earlier location which is thereby considered one of the anchors of the consistent cut. In contrast, delivering a signal arbitrarily later is likely to cause replay divergence (\$6.2). Instead, RACEPRO only considers delivering a signal later if it interrupted a system call in the recorded execution, in which case the signal is instead delivered promptly after the corresponding system call completes when replayed.

Reordering of the anchor system calls may also imply reordering of additional system calls that also access the same resources. Consider the execution scenario depicted in Figure 9, which involves three processes and five system calls that access the same resource. The system calls $S_{1}$ and $S_{5}$ form a load-store race. To generate the modified execution for this race, RACEPRO will make the following changes: (1) it will include $S_{1}$ but not $S_{2}$, because system calls following the anchors remain outside the cut and are truncated; (2) it will reorder $S_{5}$, and therefore $S_{4}$ too, with respect to $S_{1}$; and (3) depending on the consistent cut, it will either exclude $S_{3}$ or reorder $S_{3}$ with respect to $S_{1}$. RACEPRO adjusts the modified recording so that it will enforce the new partial order of system calls instead of the partial order of system calls in the original execution.

\subsection{Replaying Execution Branches and Going Live}

RACEPRO's replayer provides deterministic replay of the originally recorded execution and also ensures that successful replay of a modified execution is also deterministic. Given a modified execution, RACEPRO replays each recorded event while preserving the partial order indicated by the recording. The last events replayed are the anchor system calls. To force races to resolve as desired, RACEPRO replays the anchor system calls serially, one by one, while holding the remaining processes inactive. From that point onward, it allows the processes to go live to resume normal execution.

Go Live. The ability to go live by resuming live execution from a replay is fundamental for allowing RACEPRO to validate whether 


\begin{tabular}{lccc}
$/ /$ & $\mathbf{P}_{\mathbf{1}}$ & $\mathbf{P}_{\mathbf{2}}$ & $\mathbf{P}_{\mathbf{3}}$ \\
\cline { 2 - 4 }$/ /$ & $\ldots$ & \\
S1: & syscall(R); & & \\
S2: & syscall(R); & & $\ldots$ \\
S3: & $\ldots$ & $\ldots$ & syscall(R); \\
S4: & & syscall(R) & $\ldots$ \\
S5: & & syscall(R) & \\
& & $\ldots$ &
\end{tabular}

Figure 9: Replay divergence due to reordering.

races manifest into real bugs or not, and thereby avoid reporting false-positives. To go live, RACEPRO faces two challenges. First, RACEPRO must ensure that replayed processes perceive the underlying system to be the same as at the time of recording. For example, system identifiers such as process IDs must remain the same for processes to run correctly after they transition to live execution. RACEPRO leverages OS virtualization to encapsulate processes in a virtual execution environment that provides the same private, virtualized view of the system when the session is replayed or goes live as when it was recorded [17]. Processes only see virtual identifiers that always stay the same so that the session can go live at any time. Second, RACEPRO needs to not only replay the application state in user-space, but also the corresponding state that is internally maintained by the operating system for the processes. For example, actions such as creating a pipe and writing to it must be done as is so that the pipe exists and has suitable state should the process transition to live execution.

RACEPRO works best when a go-live execution requests no inputs from users or external processes; such executions include parallel make, parallel boot, and executions of non-interactive programs. If a go-live execution requests external inputs, RACEPRO tries to replay the inputs recorded from the original execution. Currently RACEPRO replays standard inputs from users and pipe or socket data received from external processes. It does not replay data read from the file system. Instead, it checkpoints the file system before recording an execution and restores to this checkpoint before each replay, using unionfs [29], which has low overhead. Replaying inputs may not always work because the go-live execution differs from the original execution, but we have not found it a problem in our evaluation because tightly coupled processes should be recorded together anyway.

RACEPRO can be applied recursively to detect races involving more system calls (\$5.3.4). Since it already records the go-live portion of modified executions, doing so is as easy as running the same detection logic on these new recordings. This essentially turns RACEPRO into a model checker [12]. However, we leave this mode off by default because exhaustive model checking is quite expensive and it is probably more desirable to spend limited checking resources on real executions over the fake checking-generated executions.

Replay Divergence. RACEPRO's replayer may not be able to replay some execution branches due to replay divergence. This can result from trying to replay a modified recording instead of the original recording. Replay divergence occurs when there is a mismatch between the actual actions of a replayed process and what is scripted in the execution recording. The mismatch could be between the actual system call and the expected system call or, even if the system calls match, between the resources actually accessed by the system call and the resources expected to be accessed. When a divergence failure occurs for some execution branch, RACEPRO does not flag the corresponding race as a bug because it lacks evidence to that end.

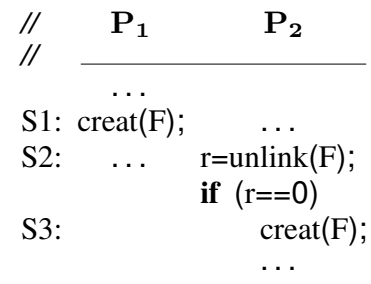

(a)

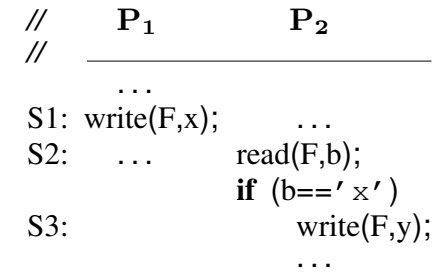

(b)
Figure 10: Replay divergence examples.

Divergence is commonly caused when the reordering of the anchor system calls implies reordering of additional system calls that also access the same resources. Consider again the execution scenario depicted in Figure 9 in which the system calls $S_{1}$ and $S_{5}$ form a load-store race and the modified execution branch reorders the systems calls as $S_{3}, S_{4}, S_{5}$, and $S_{1}$ while dropping $S_{2}$ as being outside the cut. A replay divergence may occur if the execution of $S_{5}$ depended on $S_{2}$ which was dropped out, or if the execution of $S_{4}$ depends on $S_{1}$ which was reordered with respect to $S_{4}$. Figure 10a illustrates the former scenario. Reordering the two creat system calls would cause $P_{2}$ to call unlink before $P_{1}$ 's creat. The call will fail and $P_{2}$ will not call creat and thus diverge from the recorded execution.

Divergence can also be caused when processes rely on a specific execution ordering of system calls in a way that is not tracked by RACEPRO. Figure 10b illustrates one such scenario where process $P_{1}$ executes system call $S_{1}$ to write data to a file, and process $P_{2}$ 's execution depends on data read from file by $S_{2}$. If $P_{2}$ depends on the specific data written by $S_{1}$, then reordering $S_{1}$ and $S_{2}$ will almost certainly cause a divergence. Were the dependency on the file's content considered an inherent happens-before $S_{1} \rightarrow S_{2}$, RACEPRO's explorer would not have flagged the race in the first place. However, it is prohibitively expensive, and in some cases impossible, to track generic semantics of applications.

Another cause for divergence is use of shared memory. Recall that shared memory accesses are tracked by the recorder and enforced by the replayer. However, reordering of system calls may lead to reordering of shared memory accesses as well, which will certainly lead to replay divergence. RACEPRO mitigates this effect by permitting relaxed execution from where the reordering takes place. In this mode the replayer does not enforce memory access ordering, but continues to enforce other ordering constraints such as partial ordering of system calls. This improves the chances that the replayed execution reach the point of go-live. However, accesses to shared memory may now resolve arbitrarily and still cause divergence. For this reason RACEPRO is likely to be less effective in finding races on OS resources between threads of the same process. We believe that such races are relatively unlikely to occur.

Replay divergence is reportedly a serious problem for a previous race classifier [25], where it can occur for two reasons: the race being validated does occur and causes the execution to run code or access data not recorded originally, or the race being validated cannot occur and is a false positive. In contrast, replay divergence actually helps RACEPRO to distinguish root-cause races from other races. By relying on a replay followed by transition to live execution, RACEPRO is no longer concerned with the first scenario. If replay diverges, RACEPRO can tell that the race is a false positive and discard it.

Moreover, if the divergence is not due to untracked interactions or shared memory discussed above (or file locking, also untracked by RACEPRO), then there must exist another race that is "tighter" than the one being validated. The other race may involve the same 


\section{Bug ID Description}

debian-294579 concurrent adduser processes read and write /etc/passwd without synchronization, corrupting this file

debian-438076 mv unlinks the target file before calling atomic rename, violating the atomicity requirement on mv

debian-399930 logrotate creates a new file then sets it writable, but deamons may observe it without write permissions

redhat-54127 ps I grep race causes a wrong version of licq 7.3 to be started

launchpad-596064 upstart does not wait until smbd creates a directory before spawning nmbd, which requires that directory

launchpad-10809 bash updates the history file without synchronization, corrupting this file

new-1 tcsh 6.17 updates the history file without synchronization, even when "savehist merge" is set

new-2 updatedb removes old database before renaming the new one, so locate finds nothing (findutils 4.4.2)

new-3 concurrent updatedb processes may cause the database to be empty

new-4 incorrect dependencies in Makefile of abr2gbr 1.0.3 may causes compilation failure

Table 4: Bugs found by RACEPRO. Bugs are identified by “distribution - bug ID”. New bugs are identified as "new - bug number”

resource or a different one. For example, in Figure 10b the race between $S_{1}$ and $S_{3}$ causes divergence because of another race between $S_{1}$ and $S_{2}$. The latter race is "tighter" in the sense that $S_{2}$ is closer to $S_{1}$ because $S_{2} \rightarrow S_{3}$; the race between $S_{1}$ and $S_{2}$ subsumes the race between $S_{1}$ and $S_{3}$. In other words, discarding races that cause replay divergence helps RACEPRO to find rootcause races. We believe the go-live mechanism can benefit existing replay-based thread-race classifiers.

\subsection{Checking Execution Branches}

When the replay of an execution branch switches to live execution, RACEPRO no longer controls the execution. Rather, it records the execution from that point on, and activates a checker to monitor the execution for failures or incorrect behavior. If the checker detects a failure that did not occur during recording, it reports a bug and saves the combined execution recording, consisting of the original recording followed by the new recording, so that users can deterministically replay it for debugging.

RACEPRO provides a set of built-in checkers to detect bad application behavior. The built-in checker can detect erroneous behavior such as segmentation faults, infinite loops (via timeouts), error messages in system logs, and failed commands with non-zero exit status. In addition, RACEPRO can also run system-provided checker programs such as fsck.

Moreover, RACEPRO allows users to plug in domain-specific checkers. To do so, a user need only provide a program or even a shell script that will run concurrently along the live execution. For instance, such scripts could compare the output produced by a modified execution to that of the original execution, and flag significant differences as errors. It is also possible to use existing test-suites already provided with many application packages. These test-suites are particularly handy if the target application is a server. For instance, both the Apache web server and the MySQL database server are shipped with basic though useful test suites, which could be executed against a modified server. Finally it may also compare the output of the go-live execution with a linearized run [13].

By running checkers on live executions, RACEPRO guarantees that observed failures always correspond to real executions, thus eliminating false positives if the checkers are accurate. Moreover, the process races RACEPRO detects are often the root cause of the failures, aiding developers in diagnosis. In rare cases, after a modified execution goes live, it may encounter an unrelated bug. RACEPRO still provides an execution recording useful for debugging, but without pointing out the root-cause.

As in many other checking frameworks, RACEPRO can detect only what is checked. Although its built-in checkers can detect many errors (\$7.1), it may miss domain-specific "silent" corruptions. Fortunately, recent work has developed techniques to check advanced properties such as conflict serializability or linearizability [13], which RACEPRO can leverage.
RACEPRO may have false negatives. A main source is that RACEPRO is a dynamic tool, thus it may miss bugs in the executions that do not occur. Fortunately, by checking deployed systems, RACEPRO increases its checking coverage. A second source is checker inaccuracy. If a checker is too permissive or no checker is provided to check for certain failures, RACEPRO would miss bugs.

\section{Experimental Results}

We have implemented a RACEPRO prototype in Linux. The prototype consists of Linux kernel components for record, replay, and go-live, and a Python user-space exploration engine for detecting and validating races. The current prototype has several limitations. For replaying executions and isolating the side effects of replay, RACEPRO must checkpoint system states. It currently checkpoints only file system states, though switching to better checkpoint mechanism [27] is straightforward. RACEPRO detects idle state simply by reading /proc/loadavg, and can benefit from a more sophisticated idle detection algorithm [34].

Using the RACEPRO prototype, we demonstrated its functionality in finding known and unknown bugs, and measured its performance overhead. For our experiments, the software used for RACEPRO was Linux kernel 2.6.35, Python 2.6.6, Cython 0.14, Networkx 1.1-2, and UnionFs-Fuse 0.23.

\subsection{Bugs Found}

We evaluated RACEPRO's effectiveness by testing to see if it could find both known and unknown bugs. To find known bugs, we used RACEPRO on 6 bugs from our study. Bugs were selected based on whether we could find and compile the right version of the software and run it with RACEPRO. Some of the bugs in $\$ 2$ are in programs that we cannot compile, so we excluded them from the experiments. For each known bug, we wrote a shell script to perform the operations described in the bug report, without applying any stress to make the bug easily occur. We ran this shell script without RACEPRO 50 times, and observed that the bug never occurred. We then ran RACEPRO with the script to detect the bug.

To find unknown bugs, we used four commonly used applications. We applied RACEPRO to the locate utility and updatedb, a utility to create a database for locate. These two utilities are commonly used and well tested, and they touch a shared database of file names, thus they are likely to race with each other. Inspired by the history file race in bash, we applied RACEPRO to tcsh. tcsh has a "savehist merge" option, which should supposedly merge history files from different windows and sessions. Because compilation of software packages often involves multiple concurrent and interdependent processes, we also applied RACEPRO to the make $-j$ command.

Table 4 shows all the bugs RACEPRO found. RACEPRO found a total of 10 bugs, including all of the known bugs selected and 4 previously unknown bugs. We highlight a few interesting bugs. Of the 


\begin{tabular}{|c|c|c|c|c|c|c|c|c|c|c|c|}
\hline \multirow[b]{2}{*}{ Name } & \multicolumn{3}{|c|}{ Statistics } & \multicolumn{4}{|c|}{ Number of Races } & \multicolumn{4}{|c|}{ Execution Times [seconds/race] } \\
\hline & Processes & Syscalls & Resources & Detected & Diverged & Benign & Harmful & Record & Replay & Generate & Validate \\
\hline debian-294579 & 19 & 5275 & 658 & 4232 & 3019 & 1171 & 42 & 2.47 & 2.43 & 3.42 & 2.92 \\
\hline debian-438076 & 21 & 1688 & 213 & 50 & 0 & 46 & 4 & 3.76 & 0.75 & 0.84 & 2.87 \\
\hline debian-399930 & 10 & 1536 & 279 & 17 & 0 & 13 & 4 & 0.59 & 0.57 & 0.75 & 0.84 \\
\hline redhat-54127 & 14 & 1298 & 229 & 35 & 15 & 16 & 4 & 0.27 & 0.25 & 0.66 & 0.41 \\
\hline launchpad-596064 & 34 & 5564 & 722 & 272 & 267 & 3 & 2 & 21.45 & 3.11 & 2.49 & 1.70 \\
\hline launchpad-10809 & 13 & 1890 & 205 & 143 & 117 & 16 & 10 & 0.27 & 0.25 & 0.81 & 0.44 \\
\hline new-1 & 12 & 2569 & 201 & 137 & 90 & 33 & 14 & 0.56 & 0.54 & 1.52 & 0.76 \\
\hline new-2 & 47 & 2621 & 467 & 82 & 13 & 27 & 42 & 0.89 & 0.88 & 1.44 & 1.16 \\
\hline new-3 & 30 & 4361 & 2981 & 17 & 0 & 13 & 4 & 2.63 & 2.61 & 2.34 & 2.98 \\
\hline new-4 & 19 & 4672 & 716 & 8 & 0 & 7 & 1 & 1.01 & 0.98 & 4.81 & 1.35 \\
\hline
\end{tabular}

Table 5: Bug detection statistics. Processes is the number of processes, Syscalls the number of system calls occured, and Resources the number of distinct shared resources tracked in the recorded executions. For races, Detected is the number of races detected by RACEPRO, Diverged the races for which the replay diverged (i.e., false positive), Benign the benign races, and Harmful harmful races that led to failures. Record and Replay are the times to record and replay the executions, respectively. Generate is the average time to generate an execution branch and Validate the average time to validate a race.

known bugs, the debian-294579 bug is the most serious: it leads to corruption of /etc/passwd since adduser does not synchronize concurrent reads and writes of /etc/passwd. This bug was triggered when an administrator tried to import users from OpenLDAP to a local machine.

The redhat-54127 bug is due to the ps I grep X race. Instant messenger program licq uses ps | grep to detect whether KDE or Gnome is running. Due to the race in ps I grep, licq sometimes believes a windows manager is running when it in fact is not, thus loading the wrong version of licq.

The 4 previously unknown bugs were named new-1, new-2, new-3, and new-4. In the new-1 bug, RACEPRO found that tcsh writes to its history file without proper synchronization, even when "savehist merge" is set. This option is supposed to merge history across windows and sessions, but unfortunately, it is not implemented correctly.

In the new- 2 bug, RACEPRO found that when locate and updatedb run concurrently, locate may observe an empty database and return zero results. The reason is that updatedb unlinks the old database, before calling rename to replace it with the new database. This unlink is unnecessary as rename guarantees atomic replacement of the destination link.

In the new-3 bug, RACEPRO found that when multiple instances of updatedb run concurrently, the resultant database may be corrupted. Multiple updatedb processes may exist, for example, when users manually run one instance while cron is running another. While updatedb carefully validates the size of the new database before using it to replace the old one, the validation and replacement are not atomic, and the database may still be corrupted.

In the new-4 bug, RACEPRO found that in the compilation of abr2gbr, a package to convert between image formats, the build process may fail when using make $-j$ for parallel compilation. The reason is that the dependencies defined in the Makefile are incomplete, which produces a race condition between the creation of an \$OBJDIR directory and the use of that directory to store object files from the compilation.

\subsection{Bug Statistics}

Table 5 shows various statistics for each detected bug, including the number of processes involved (Processes), the number of system calls recorded (Syscalls), the number of unique shared resources tracked (Resources), the total number of races detected (Races), the number of races in which the replay diverged (Diverged), the number of benign races (Benign), and the number of harmful races
(Harmful). The number of processes tends to be large because when running a shell script, the shell forks a new process for each external command. The number of system calls in the recorded executions ranges from 1,298 to 5,564 . The number of distinct shared resources accessed by these system calls ranges from 201 to 2,981.

The number of races that RACEPRO detects varies across different bugs. For instance, RACEPRO detected only 17 races for debian-399930, but it detected over 4,000 races for debian-294579. Typically only a small number of races are harmful, while the majority are benign, as shown by the Benign column. In addition, RACEPRO effectively pruned many false positives as shown by the Diverged column. These two columns together illustrate the benefit of the replay and go-live approach.

The mapping between harmful races and bugs is generally manyto-one. There are multiple distinct races that produce the same or similar failures due to a common logical bug. There are two main reasons why a single programming error may result in multiple races. First, a bug may occur in a section of the code that is executed multiple times, for instance in a loop, or in a function called from multiple sites. Thus, there can be multiple races involving distinct instances of the same resource type; RACEPRO will detect and validate each independently. Second, a bug such as missing locks around critical sections may incorrectly allow reordering of more than two system calls, and each pair of reordered system calls could produce a distinct race.

In most cases, we relied on built-in checkers in RACEPRO to detect the failures. For instance, RACEPRO caught bug launchpad596064 by using grep to find error messages in standard daemon logs, and it caught bugs debian-438076, debian-399930, new-2, new-3, and new-4 by checking for the exit status of programs. Writing checkers to detect other cases was also easy, and required just one line in all cases. For example, for debian-294579, launchpad10809, and new-1, we detected the failures simply using a diff of the old and new versions of the affected file.

\subsection{Performance Overhead}

Low recording overhead is crucial because RACEPRO runs with deployed systems. Low replay overhead is desirable because RACEPRO can check more execution branches within the same amount of time. To evaluate RACEPRO's record and replay overhead, we applied it to a wide range of real applications on an IBM HS20 eServer BladeCenter, each blade with dual $3.06 \mathrm{GHz}$ Intel Xeon CPUs with hyperthreading, 2.5 GB RAM, a 40 GB local disk, interconnected with a Gigabit Ethernet switch. These applications include 
(1) server applications such as Apache in multi-process and multithreaded configurations, MySQL, an OpenSSH server, (2) utility programs such as SSH clients, make, untar, compression programs such as gzip and lzma, and a vi editor, and (3) graphical desktop applications such as Firefox, Acrobat Reader, MPlayer, and OpenOffice. To run the graphical applications on the blade which lacks a monitor, we used VNC to provide a virtual desktop. For application workloads that required clients and a server, we ran the clients on one blade and the server on another. Our results show that RACEPRO's recording overhead was under $2.5 \%$ for server and under $15 \%$ for desktop applications. Replay speed was in all cases at least as fast as native execution and in some cases up to two orders of magnitude faster. This speedup is particularly useful for enabling rapid race validation. Replay speedup stems from omitted in-kernel work due to system calls partially or entirely skipped, and waiting time skipped at replay. Applications that do neither operations perform the same work whether recording or replaying, and sustain speedups close to 1 .

We also measured various overhead statistics involved in finding the bugs listed in Table 5. These measurements were done on an HP DL360 G3 server with dual $3.06 \mathrm{GHz}$ Intel Xeon CPUs, 4 GB RAM, and dual 18 GB local disks. For each bug, Table 5 shows the time to record the execution (Record) and to replay it (Replay), the average time to generate an execution branch for a race from a recorded execution (Generate), and the average time to validate an execution branch for a race (Validate).

In all cases, recording execution times were within $3 \%$ of the original execution times without recording, and replaying the execution took less time than the original recorded execution. Replay time for each recording ranged from $250 \mathrm{~ms}$ to $1.8 \mathrm{~s}$, providing an upper limit on the time to replay execution branches. Replaying execution branches is generally faster because those branches are truncated versions of the original execution. Replay speedup was near 1 in most cases, but was as high as 7 times for launchpad596064 due to very long idle times as part of starting up the workload. These results are in line with our other record-replay results for desktop and server applications. In particular, the results demonstrate that RACEPRO recording overhead is low enough to enable its use on deployed systems.

The time for our unoptimized prototype to detect all races was under $350 \mathrm{~ms}$ for most bugs, but in some cases as much as $3.8 \mathrm{~s}$. This time correlates roughly with the number of unique shared kernel objects tracked and the number of processes involved. For example, detecting all races for launchpad-596064 took $2.5 \mathrm{~s}$, or less than $0.5 \mathrm{~ms}$ per race. The average time to generate an execution branch for a race ranged from $0.66 \mathrm{~s}$ to $4.81 \mathrm{~s}$. This time correlates roughly with the number of system calls. The average time to validate a race ranged from $0.44 \mathrm{~s}$ to $2.98 \mathrm{~s}$. This time correlates roughly with the replay time.

In most cases, the average time to validate a race was somewhat larger than the time to replay the original execution by $0.3 \mathrm{~s}$ to $2 \mathrm{~s}$. The time to validate a race is longer because, in addition to the time to replay the execution branch, it also includes the time to run the go-live execution, run the checker, and perform setup and cleanup work between races. Replaying an execution branch which ends at the anchor system calls is faster than replaying the whole original execution. However, during validation, the remainder of the recorded execution now runs live, which is usually slower than replayed execution. In one case, launchpad-596064, validation was faster then original execution replay because nearly all of the execution branches resulted in replay divergence relatively early, eliminating the additional time it would take to replay the entire execution branches and have them go live.
The Generate and Validate times are averaged per race, so the total time to generate execution branches and validate races will grow with the number of races. However, races are independent of one another, so these operations can be easily done in parallel on multiple machines to speed them up significantly. Overall, the results show that RACEPRO can detect harmful process races not only automatically without human intervention, but efficiently.

\section{Related Work}

We previously presented in a workshop paper [16] a preliminary design of RACEPRO, without the full design, implementation, and evaluation described in this paper. In the remainder of this section, we discuss closely related work to RACEPRO.

Thread races. Enormous work has been devoted to detecting, diagnosing, avoiding, and repairing thread races (e.g., [11, 24, 25, 30, $36,38])$. However, as discussed in $\S 1$, existing systems for detecting thread races do not directly address the challenges of detecting process races. For instance, existing static race detectors work with programs written in only one language $[11,24]$; the dynamic ones detect races within only one process and often incur high overhead (e.g., [23]). In addition, no previous detection algorithms as we know of explicitly detect wait-wakeup races, a common type of process races.

Nonetheless, many ideas in these systems apply to process races once RACEPRO models system call effects as load and store micro-operations. For instance, we may leverage the algorithm in AVIO [21] to detect atomicity violations involving multiple processes; the consequence-oriented method in ConSeq [40] to guide the detection of process races; and serializability or linearizability checking [13].

A recent system, 2ndStrike [14], detects races that violate complex access order constraints by tracking the typestate of each shared object. For instance, after a thread calls close (fd), 2ndStrike transits the file descriptor to a "closed" state; when another thread calls read(fd), 2ndStrike flags an error because reads are allowed only on "open" file descriptors. RACEPRO may borrow this idea to model system calls with richer effects, but we have not found the need to do so for the bugs RACEPRO caught.

RACEPRO leverages the replay-classification idea [25] to distill harmful races from false or benign ones. The go-live mechanism in RACEPRO improves on existing work by turning a replayed execution into a real one, thus avoiding replay divergence when a race does occur and changes the execution to run code not recorded.

We anticipate that ideas in RACEPRO can help thread race detection, too. For instance, thread wait and wakeup operations may also pair up in different ways, such as a sem_post waking up multiple sem_down calls. Similarly, the go-live mechanism can enable other race classifiers to find "root races" instead of derived ones.

TOCTOU races. TOCTOU race detection $[32,33,35]$ has been a hot topic in the security community. Similar to RACEPRO, these systems often perform OS-level detection because file accesses are sanitized by the kernel. However, тостоU races often refer to specific types of races that allow an attacker to access unauthorized files bypassing permission checks. In contrast, RACEPRO focuses on general process races and resources not only files. Nonetheless, RACEPRO can be used to detect TOCTOU races in-vivo, which we leave for future work.

Checking deployed systems. Several tools can also check deployed systems. CrystalBall [37] detects and avoids errors in a deployed distributed system using an efficient global state collection and exploration technique. Porting CrystalBall to detect process races is difficult because it works only with programs written in a special language, and it does checking while the deployed system 
is running, relying on network delay to hide the checking overhead. In-vivo testing [8] uses live program states, but it focuses on unit testing and lacks concurrency support.

To reduce the overhead on a deployed system, several systems decouple execution recording from dynamic analysis [7, 26]. RACEPRO leverages this approach to check process races. One difference is that RACEPRO uses OS-level record and replay, which has lower overhead than [7] and, unlike Speck [26], RACEPRO works with both multiprocess and multithreaded applications. In addition, a key mechanism required for validating races is that RACEPRO can faithfully replay an execution and make it go-live at any point, which neither previous system can do.

OS support for determinism and transaction. Our idea to pervasively detect process races is inspired by operating system transactions in TxOS [28] and pervasive determinism in Determinator [5] and dOS [6]. TxOS provides transaction support for heterogeneous OS resources, efficiently and consistently solving many concurrency problems at the OS level. For instance, it can prevent file system TOCTOU attacks. However, as pointed out in [20], even with transaction support, execution order violations may still occur. Determinator advocates a new, radical programming model that converts all races, including thread and process races, into exceptions. A program conforming to this model runs deterministically in Determinator. dOS makes legacy multithreaded programs deterministic even in the presence of races on memory and other shared resources. None of these systems aim to detect process races.

\section{Conclusion and Future Work}

We have presented the first study of real process races, and the first system, RACEPRO, for effectively detecting process races beyond TOCTOU and signal races. Our study has shown that process races are numerous, elusive, and a real threat. To address this problem, RACEPRO automatically detects process races, checking deployed systems in-vivo by recording live executions and then checking them later. It thus increases checking coverage beyond the configurations or executions covered by software vendors or beta testing sites. First, RACEPRO records executions of multiple processes while tracking accesses to shared kernel resources via system calls. Second, it detects process races by modeling recorded system calls as load and store micro-operations to shared resources and leveraging existing memory race detection algorithms. Third, for each detected race, it modifies the original recorded execution to reproduce the race by changing the order of system calls involved in the races. It replays the modified recording up to the race, allows it to resume live execution, and checks for failures to determine if the race is harmful. We have implemented RACEPRO, shown that it has low recording overhead so that it can be used with minimal impact on deployed systems, and used it with real applications to effectively detect 10 process races, including several previously unknown bugs in shells, databases, and makefiles.

Detection of process races is only the first step. Given an execution where a process race surfaces, developers still have to figure out the cause of the race. Fixing process races take time, and before developers produce a fix, systems remain vulnerable. Exploring the possibility of automatically fixing process races and providing better operating system primitives to eliminate process races are important areas of future work.

\section{Acknowledgements}

Our shepherd Tim Harris and the anonymous reviewers provided many helpful comments, which have substantially improved the content and presentation of this paper. Peter Du helped with the process race study. Dawson Engler provided early feedback on the ideas of this paper. This work was supported in part by AFRL FA8650-10-C-7024 and FA8750-10-2-0253, AFOSR MURI FA9550-07-1-0527, and NSF grants CNS-1117805, CNS-1054906 (CAREER), CNS-1012633, CNS-0914845, and CNS-0905246.

\section{References}

[1] All resource races studied. http: //rcs.cs.columbia.edu/projects/racepro/.

[2] Launchpad Software Collaboration Platform. https: // launchpad.net/.

[3] The Debian Almquist Shell. http: //gondor.apana.org.au/ herbert/dash/.

[4] Upstart: an Event-Based Replacement for System V Init Scripts. http: / / upstart. ubuntu.com/.

[5] A. Aviram, S.-C. Weng, S. Hu, and B. Ford. Efficient System-Enforced Deterministic Parallelism. In Proceedings of the 9th Symposium on Operating Systems Design and Implementation (OSDI'10), Oct. 2010.

[6] T. Bergan, N. Hunt, L. Ceze, and S. D. Gribble. Deterministic Process Groups in dOS. In Proceedings of the 9th Symposium on Operating Systems Design and Implementation (OSDI '10), Oct. 2010.

[7] J. Chow, T. Garfinkel, and P. M. Chen. Decoupling Dynamic Program Analysis from Execution in Virtual Environments. In Proceedings of the USENIX Annual Technical Conference (USENIX '08), June 2008.

[8] M. Chu, C. Murphy, and G. Kaiser. Distributed In Vivo Testing of Software Applications. In Proceedings of the First IEEE International Conference on Software Testing, Verification, and Validation (ICST'08), Apr. 2008.

[9] H. Cui, J. Wu, C.-C. Tsai, and J. Yang. Stable Deterministic Multithreading through Schedule Memoization. In Proceedings of the 9th Symposium on Operating Systems Design and Implementation (OSDI '10), Oct. 2010.

[10] G. W. Dunlap, D. G. Lucchetti, M. A. Fetterman, and P. M. Chen. Execution Replay of Multiprocessor Virtual Machines. In Proceedings of the 4th International Conference on Virtual Execution Environments (VEE '08), Mar. 2008.

[11] D. Engler and K. Ashcraft. RacerX: Effective, Static Detection of Race Conditions and Deadlocks. In Proceedings of the 19th ACM Symposium on Operating Systems Principles (SOSP '03), Oct. 2003.

[12] C. Flanagan and P. Godefroid. Dynamic Partial-Order Reduction for Model Checking Software. In Proceedings of the 32nd Annual Symposium on Principles of Programming Languages (POPL '05), Jan. 2005.

[13] P. Fonseca, C. Li, and R. Rodrigues. Finding Complex Concurrency Bugs in Large Multi-Threaded Applications. In Proceedings of the 6th ACM European Conference on Computer Systems (EUROSYS '11), Apr. 2011.

[14] Q. Gao, W. Zhang, Z. Chen, M. Zheng, and F. Qin. 2ndStrike: Towards Manifesting Hidden Concurrency Typestate Bugs. In Proceedings of the 16th International Conference on Architecture Support for Programming Languages and Operating Systems (ASPLOS '11), Mar. 2011.

[15] Z. Guo, X. Wang, J. Tang, X. Liu, Z. Xu, M. Wu, M. F. Kaashoek, and Z. Zhang. R2: An Application-Level Kernel for Record and Replay. In Proceedings of the 8th Symposium on Operating Systems Design and Implementation (OSDI '08), Dec. 2008.

[16] O. Laadan, C.-C. Tsai, N. Viennot, C. Blinn, P. S. Du, 
J. Yang, and J. Nieh. Finding Concurrency Errors in Sequential Code-OS-level, In-vivo Model Checking of Process Races. In Proceedings of the 13th USENIX Workshop on Hot Topics in Operating Systems (HOTOS '11), May 2011.

[17] O. Laadan, N. Viennot, and J. Nieh. Transparent, Lightweight Application Execution Replay on Commodity Multiprocessor Operating Systems. In Proceedings of the ACM

SIGMETRICS Conference on Measurement and Modeling of Computer Systems (SIGMETRICS '10), June 2010.

[18] L. Lamport. Time, Clocks, and the Ordering of Events in a Distributed System. Comm. ACM, 21(7):558-565, 1978.

[19] T. J. LeBlanc and J. M. Mellor-Crummey. Debugging Parallel Programs with Instant Replay. IEEE Trans. Comput., 36(4):471-482, 1987.

[20] S. Lu, S. Park, E. Seo, and Y. Zhou. Learning from Mistakes: a Comprehensive Study on Real World Concurrency Bug Characteristics. In Proceedings of the 13th International Conference on Architecture Support for Programming Languages and Operating Systems (ASPLOS '08), Mar. 2008.

[21] S. Lu, J. Tucek, F. Qin, and Y. Zhou. AVIO: Detecting Atomicity Violations via Access Interleaving Invariants. In Proceedings of the 12th International Conference on Architecture Support for Programming Languages and Operating Systems (ASPLOS '06), Oct. 2006.

[22] F. Mattern. Dynamic Partial-Order Reduction for Model Checking Software. In Proceedings of the 32nd Annual Symposium on Principles of Programming Languages (POPL '05). Oct. 1988.

[23] M. Musuvathi, S. Qadeer, T. Ball, G. Basler, P. A. Nainar, and I. Neamtiu. Finding and Reproducing Heisenbugs in Concurrent Programs. In Proceedings of the 8th Symposium on Operating Systems Design and Implementation (OSDI '08), Dec. 2008.

[24] M. Naik, A. Aiken, and J. Whaley. Effective Static Race Detection For Java. In Proceedings of the ACM SIGPLAN 2006 Conference on Programming Language Design and Implementation (PLDI '06), 2006.

[25] S. Narayanasamy, Z. Wang, J. Tigani, A. Edwards, and B. Calder. Automatically Classifying Benign and Harmful Data Racesallusing Replay Analysis. In Proceedings of the ACM SIGPLAN 2007 Conference on Programming Language Design and Implementation (PLDI '07), June 2007.

[26] E. B. Nightingale, D. Peek, P. M. Chen, and J. Flinn. Parallelizing Security Checks on Commodity Hardware. In Proceedings of the 13th International Conference on Architecture Support for Programming Languages and Operating Systems (ASPLOS '08), Mar. 2008.

[27] S. Osman, D. Subhraveti, G. Su, and J. Nieh. The Design and Implementation of Zap: A System for Migrating Computing Environments. In Proceedings of the 5th Symposium on Operating Systems Design and Implementation (OSDI '02), Dec. 2002.

[28] D. E. Porter, O. S. Hofmann, C. J. Rossbach, A. Benn, and E. Witchel. Operating System Transactions. In Proceedings of the 22nd ACM Symposium on Operating Systems Principles (SOSP '09), Oct. 2009.

[29] D. P. Quigley, J. Sipek, C. P. Wright, and E. Zadok. UnionFS: User- and Community-oriented Development of a Unification Filesystem. In Proceedings of the 2006 Linux Symposium, July 2006.

[30] K. Sen. Race Directed Random Testing of Concurrent
Programs. In Proceedings of the ACM SIGPLAN 2008 Conference on Programming Language Design and Implementation (PLDI '08), June 2008.

[31] S. M. Srinivasan, S. Kandula, C. R. Andrews, and Y. Zhou. Flashback: A Lightweight Extension for Rollback and Deterministic Replay for Software Debugging. In Proceedings of the USENIX Annual Technical Conference (USENIX '04), June 2004.

[32] D. Tsafrir, T. Hertz, D. Wagner, and D. Da Silva. Portably Solving File TOCTTOU Races with Hardness Amplification. In Proceedings of the 6th USENIX Conference on File and Storage Technologies (FAST '08), Feb. 2008.

[33] E. Tsyrklevich and B. Yee. Dynamic Detection and Prevention of Race Conditions in File Accesses. In Proceedings of the 12th Conference on USENIX Security Symposium, Aug. 2003.

[34] University of California at Berkeley. Open-Source Software for Volunteer Computing and Grid Computing. http: //boinc.berkeley.edu/.

[35] J. Wei and C. Pu. TOCTTOU Vulnerabilities in UNIX-Style File Systems: an Anatomical Study. In Proceedings of the 4th USENIX Conference on File and Storage Technologies (FAST '05), Dec. 2005.

[36] J. Wu, H. Cui, and J. Yang. Bypassing Races in Live Applications with Execution Filters. In Proceedings of the 9th Symposium on Operating Systems Design and Implementation (OSDI '10), Oct. 2010.

[37] M. Yabandeh, N. Knezevic, D. Kostic, and V. Kuncak. CrystalBall: Predicting and Preventing Inconsistencies in Deployed Distributed Systems. In Proceedings of the 6th Symposium on Networked Systems Design and Implementation (NSDI '09), Apr. 2009.

[38] Y. Yu, T. Rodeheffer, and W. Chen. RaceTrack: Efficient Detection of Data Race Conditions via Adaptive Tracking. In Proceedings of the 20th ACM Symposium on Operating Systems Principles (SOSP '05), Oct. 2005.

[39] M. Zalewski. Delivering Signals for Fun and Profit. Bindview Corporation, 2001.

[40] W. Zhang, J. Lim, R. Olichandran, J. Scherpelz, G. Jin, $\mathrm{S}$. Lu, and T. Reps. ConSeq: Detecting Concurrency Bugs through Sequential Errors. In Proceedings of the 16th International Conference on Architecture Support for Programming Languages and Operating Systems (ASPLOS '11), Mar. 2011. 